\title{
Evaluation of Software Requirement Specification Based on IEEE 830 Quality Properties
}

\author{
E. Stephen ${ }^{\mathrm{a}, 1}$, E. Mit ${ }^{\mathrm{a}, 2}$ \\ ${ }^{a}$ Faculty of Computer Science and Information Technology,Universiti Malaysia Sarawak (UNIMAS),94300, Sarawak, Malaysia. \\ E-mail: ${ }^{1}$ ellystephen@yahoo.com; ${ }^{2}$ edwin@unimas.my
}

\begin{abstract}
Software requirement specification (SRS) documented an essential requirement of software and its external interface. Many studies found the quality of SRS, but lack of the informality organizing of document and representation of functional requirement. This paper aims to evaluate the quality properties of the software requirement specification (SRS). There are four quality properties to be assessed, which are completeness, correctness, preciseness, and consistency. Completeness quality is used to evaluate the structure of the SRS document; meanwhile, the other three qualities used to evaluate the functional requirement. The measurement for each quality properties has been proposed in the previous study. The evaluation process involves a few stages. In short, the prototype would extract text through the provided document, do a calculation, and came out with the result in the form of a similarity percentage. The prototype designs in such ways it minimizes the user interference. Those resulted in reducing human error. Corpus contains libraries of term and topic are expected to increase the reliability of detection. The corpus includes topics extracted from IEEE 830 standard, vague word, terms represent Create, Read, Update, and Delete (CRUD) operation, and terms denote possible datatype. The extracted functional requirement would be refined based on the Requirement Boilerplate (RB) template. RB adopted in the study to ensure the consistency of functional refinement requirements. The percentage of similarity is determined based on comparison with IEEE 830 standard. The rate of the result of each quality properties reflects the quality of the software requirement specification.
\end{abstract}

Keywords - quality properties; quantitative approach; requirement engineering; software requirement specification.

\section{INTRODUCTION}

Software Requirement Specification (SRS) document has become crucial for development. It acts as a guideline and medium of agreement between the client and the developer. It also used to validate the requirement with the stakeholder [1]. Due to heterogeneous software domains, this study focuses on the general properties that must be fulfilled by the domain. The general properties would reflect the quality of the SRS document itself. It is important to consider that software developer meet the quality properties as the document tend to be shared among different level of the organization [2]. Two criteria need to be considered in writing the SRS [3]. First, the SRS must be readable. In brief, the structure of the document must be organized, which lead the reviewer to view the context at ease. Second, the SRS must list a processable requirement with a degree of quality for each functional requirement are stated clearly. The SRS should be correct, complete, consistent, unambiguous, verifiable, modifiable, and traceable [4].

Nowadays, requirement engineering (RE) value increasingly important due to the complexity of capturing the requirement proposed by the client. Requirement
Boilerplate has been introduced in the SRS to overcome the complexity. Requirement Boilerplate provides the uniformity for the functional requirement. By the Requirement Boilerplate, the capturing or understanding of the functional requirement could increase. The Requirement Boilerplate is also introduced due to the ambiguity in the natural language [5]. This paper discusses the output of the system, which is built to cater to the heterogeneous SRS domain. Four types of quality properties were measured. These include completeness, consistency, correctness, and preciseness [6]. The assessment target is used to measure the structural component of the SRS and its functional requirements. As stated above, the measurement would focus on four quality properties. These include completeness, consistency, correctness, and preciseness. Completeness quality properties was targeted on the structural of the SRS; meanwhile, the other three proposed quality properties focus on the functional requirement. The framework of the proposed measurement is shown in the study before [6].

\section{A. Structural}

The completeness quality properties are used to measure the percentage of similarity between the standard structure 